\title{
Determinants of Initial Antibiotic Duration in Very Low Birth Weight Neonates
}

\author{
Alexandra C. Charron (D) - Mike A. Carl - Barbara B. Warner • \\ Jason G. Newland · Christopher C. McPherson
}

Received: January 24, 2019 / Published online: March 1, 2019

(C) The Author(s) 2019

\begin{abstract}
Introduction: Very low birth weight (VLBW) neonates $(<1500 \mathrm{~g})$ are commonly exposed to prolonged antibiotic courses related to concerns for presumed early onset sepsis often with unclear indications. While antibiotics can be life-saving medications, prolonged antibiotic exposure ( $>5$ days) increases an infant's risk for necrotizing enterocolitis, late onset sepsis, colonization or infection with resistant organisms, and death. The aim of this study is to describe clinical and laboratory factors that influence the length of initial antibiotic courses in VLBW neonates.

Methods: Demographics, perinatal factors, and neonatal clinical and laboratory data were
\end{abstract}

Enhanced digital features To view enhanced digital features for this article go to https://doi.org/10.6084/ m9.figshare.7739798.

A. C. Charron $(\varangle) \cdot$ B. B. Warner - C. C. McPherson Department of Pediatrics, Division of Newborn Medicine, Washington University School of Medicine, St. Louis, MO, USA

e-mail: alexandracharron@wustl.edu

M. A. Carl

Department of Pediatrics, Washington University

School of Medicine, St. Louis, MO, USA

J. G. Newland

Department of Pediatrics, Division of Infectious

Diseases, Washington University School of

Medicine, St. Louis, MO, USA compared in a single-center retrospective cohort of VLBW neonates who received $\leq$ 3 days versus $>5$ days of initial antibiotics.

Results: A total of 121 patients were analyzed of which 117 (97\%) were started on antibiotics empirically on admission, and 71 (59\%) received $\leq 3$ days and $50(41 \%)$ received $>$ 5 days of antibiotics. One $(0.8 \%)$ infant had a positive blood culture (S. oralis). Demographics [gestational age $(p<0.001)$ and birth weight $(p<0.001)]$ and neonatal clinical status [Apgar score at $5 \mathrm{~min}(p=0.001)$, CRIB II $(p<0.001)$, need for inotropes $(p=0.001)$, and maximum ventilator support $(p<0.001)]$ were significantly different between the short and prolonged course of antibiotics groups on bivariate analysis. There were no significant differences in perinatal factors or common laboratory markers of sepsis. Maximum ventilator support remained significant on multivariate analysis $(p=0.007)$.

Conclusion: In the VLBW population, the clinical status of the neonate, as represented by maximum ventilator support in this study, was the most important factor in determining the duration of initial antibiotic treatment. Laboratory values and perinatal risk factors did not significantly influence prescribing patterns.

Keywords: Antibiotic stewardship; Early onset sepsis; Neonatal intensive care unit; Very low birth weight infants 


\section{INTRODUCTION}

Antibiotics are the most commonly prescribed medications in a neonatal intensive care unit (NICU), particularly those agents used for early onset sepsis (EOS) [1]. Prescribing patterns of antibiotics show significant variations between hospitals as well as between different providers within the same center, with no correlation between antibiotic use rate and proven infections or mortality [2]. While infection remains a significant cause of mortality in preterm neonates, overuse of antibiotics in this population, driven primarily by prolonged antibiotic courses, is well documented $[3,4]$.

Prolonged antibiotic exposure is associated with adverse outcomes in very low birth weight (VLBW $<1500$ g) neonates. Early empiric exposure to prolonged antibiotics increases the risk for necrotizing enterocolitis, late-onset sepsis, and death $[5,6]$. Additionally, thirdgeneration cephalosporin exposure increases the risk of candida colonization and invasive candidiasis $[7,8]$. Colonization with resistant organisms is an emerging problem in neonatology, and is linked to treatment with broadspectrum antimicrobial agents [9]. Specifically, antibiotic exposure dramatically alters the preterm infant gut microbiome, enriching the presence of multidrug-resistant organisms [10]. Physicians in the NICU face the difficult task of providing potentially life-saving antibiotic treatment in appropriately selected neonates, yet limiting the associated risks to the individual patient and the overall population.

National efforts have focused on evidencebased initiation of antibiotics in term and late preterm neonates. Validated predictive models for EOS in this population use objective data combined with physical exam, and have resulted in improved antibiotic use $[11,12]$. Limited data exist to inform similar models in preterm neonates, likely secondary to the numerous challenges inherent to the clinical diagnosis of sepsis in preterm neonates. Biomarkers of sepsis are unreliable in preterm neonates, and must be used in combination with clinical judgment to diagnose sepsis [13]. Clinical signs of sepsis, such as respiratory distress and hypotension, could be the result of a number of other common co-morbidities of prematurity, including respiratory distress syndrome and patent ductus arteriosus.

Currently, VLBW neonates receive prolonged empiric antibiotic courses, often irrespective of perinatal events [14]. Understanding the key drivers of antibiotic duration in preterm neonates is essential in order to investigate the validity of these drivers and ultimately develop improved guidelines for antibiotic use in this population. The objective of this study is to describe clinical and laboratory factors that influence the length of the initial antibiotic course in VLBW neonates.

\section{METHODS}

A retrospective chart review was conducted of inborn VLBW neonates admitted to the St. Louis Children's Hospital NICU from January 1 to December 31, 2014. Neonates with major congenital malformations or death in the first 5 days of life were excluded. This study was performed in compliance with the 1964 Helsinki declaration and its later amendments or comparable ethical standards. The Institutional Review Board of Washington University in St. Louis approved this study and granted a waiver of consent given its retrospective nature.

\section{Data Collection}

Maternal and/or infant factors that have been associated with neonatal infection and other factors identified to potentially influence physician prescribing of antibiotics were collected. Demographic data including gestational age, birth weight, sex, race, ethnicity, and multiplicity of gestations were collected for each patient. Antepartum risk factors included presence of chorioamnionitis, length of rupture of membranes, route of delivery, placental abruption, preeclampsia, maternal antibiotic administration, indication for maternal antibiotic administration, maternal group B streptococcus (GBS) carrier status, and infectious concern at time of delivery. Infectious concern at delivery was defined as any indication for 
delivery having known association with infection, including preterm labor, premature rupture of membranes, chorioamnionitis, and placental abruption. Presence of chorioamnionitis was based on clinical diagnosis by the obstetric medical team, using criteria for suspected intraamniotic infection [15]. Neonatal characteristics obtained included APGAR score at 1 and $5 \mathrm{~min}$ of life, clinical risk index for babies II (CRIB II) score, blood culture results, initial white blood cell count (WBC), absolute neutrophil count (ANC), immature to total neutrophil (I:T) ratio, platelet count, maximum ventilator support in the first $48 \mathrm{~h}$ of life, and need for inotropic support in the first $48 \mathrm{~h}$ of life. Maximum ventilator support was categorized as room air or non-invasive support, conventional invasive mechanical ventilation, or high-frequency oscillator ventilation.

At our institution, there is no formal written guideline to approach EOS in VLBW neonates. During the time period of this study, blood cultures and complete blood count (CBC) were typically obtained on admission. The blood culture policy states that two cultures should be obtained from two separate sites and at least $0.5 \mathrm{~mL}$ of blood placed in each bottle. The standard antibiotic regimen for EOS is ampicillin and gentamicin, with dosing depending on the gestational age and weight of the patient.

\section{Antibiotic Data}

The duration of antibiotic administration begun in the first $72 \mathrm{~h}$ of life for the empiric treatment of suspected EOS was collected. Exposure to any antibiotic dose on a calendar day was counted as 1 day of antibiotics. Neonates who did not receive any antibiotic doses in the first $72 \mathrm{~h}$ of life were considered to have zero days of antibiotic exposure. Subgroups were formed based on antibiotic duration: short course was defined as less than or equal to 3 days of antibiotics and prolonged course was defined as greater than 5 days of antibiotics. Patients who received 4 or 5 days of antibiotics were omitted from further analysis, as the clinical intent was not clearly for a short or prolonged course at these durations.

\section{Statistical Analysis}

Clinical factors and laboratory values for the neonates who received $\leq 3$ days of antibiotics or $>5$ days of antibiotics were compared using the Mann-Whitney $U$ test and Fisher's exact test for continuous and binomial variables, respectively. Factors significantly different on univariate comparisons $(p<0.10)$ were considered for inclusion in the regression model. A multivariate logistic regression model was created with factors selected based on strength of association on univariate analysis and clinical importance. All statistics were performed using IBM SPSS Statistics Grad Pack 24.0.

\section{RESULTS}

A total of 140 VLBW neonates were inborn during the study period. Four neonates died before day of life 5, resulting in 136 neonates that met our inclusion criteria. Overall, 132 neonates $(97.5 \%)$ were empirically started on antibiotics in the first $72 \mathrm{~h}$ of life. The median initial antibiotic duration was 3 days with a range of $0-21$ days. A total of 71 neonates (52\%) received $\leq 3$ days of antibiotics, $15 \quad(11 \%)$ received 4-5 days of antibiotics, and 50 (37\%) received $>5$ days of antibiotics. Median antibiotic duration increased with decreasing gestational age (Fig. $1 ; p<0.001$ ). All neonates started on antibiotics had blood cultures obtained prior to initiation. One patient had a positive blood culture, identified as Streptococcus oralis.

Comparisons by initial antibiotic duration are presented in Table 1 . Neonates who received a prolonged antibiotic course were younger in gestational age $(p<0.001)$, of lower birthweight $(p<0.001)$, and had lower APGAR scores at 5 min of life $(p=0.001)$, and higher CRIB II scores $(p<0.001)$. Maximum ventilator support 


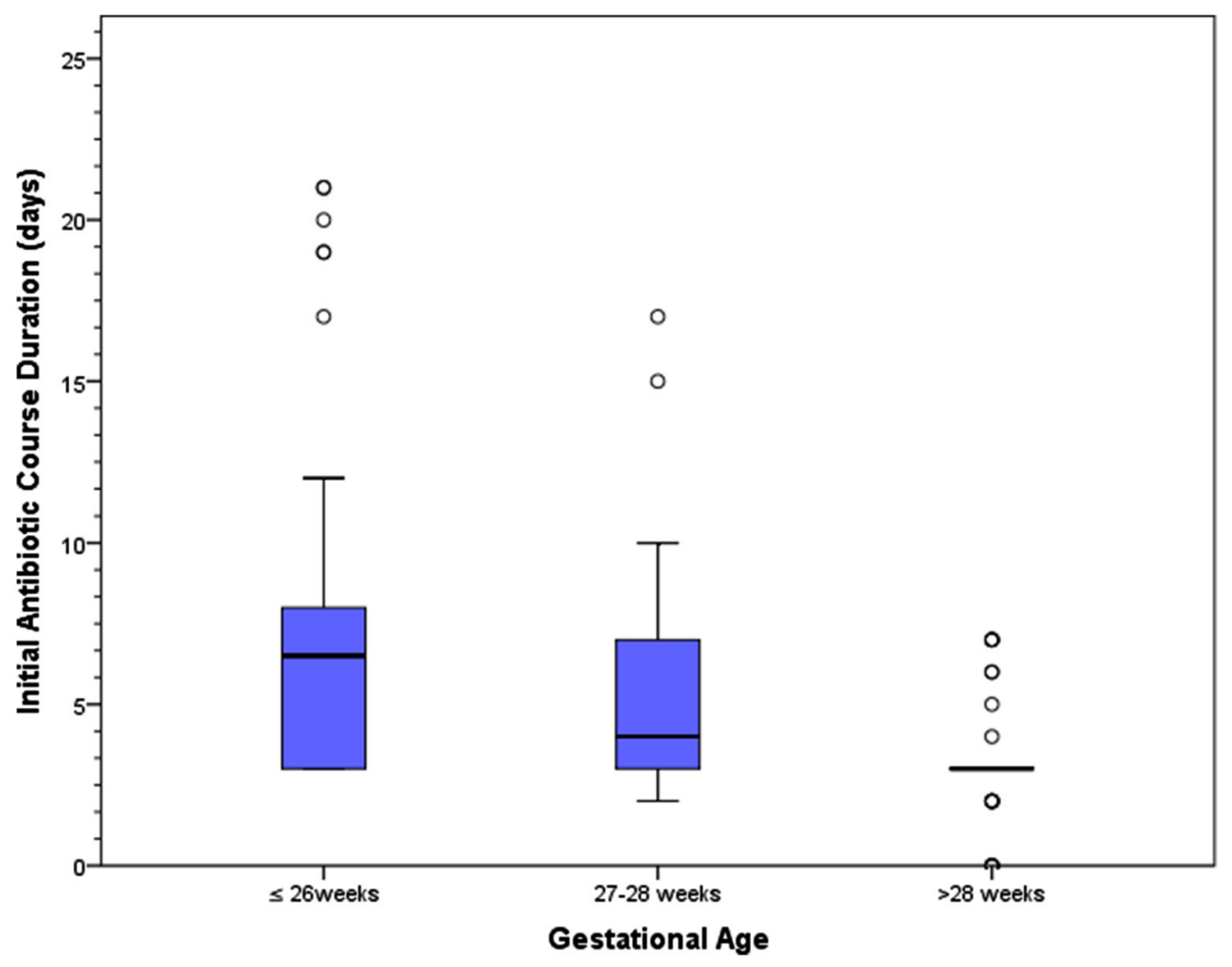

Fig. 1 Initial antibiotic course duration stratified by gestational age

$(p<0.001)$ and inotrope use in the first $48 \mathrm{~h}$ $(p=0.001)$ were significantly different between groups.

Significant collinearity was found between factors, including gestational age, birth weight, CRIB II score, APGAR scores and illness severity markers. As a result, commonly recognized maternal, demographic, perinatal, and clinical factors meeting univariate cut-off values $(p<0.1)$ were selected for the multivariate analysis, and included chorioamnionitis, gestational age, APGAR score at $5 \mathrm{~min}$ of life, and maximum ventilator support. Only maximum ventilator support significantly predicted a prolonged antibiotic course controlling for covariates (Table 2).

\section{DISCUSSION}

This single-center retrospective study demonstrates that prolonged antibiotic duration in our VLBW population was most consistently predicted by clinical illness severity as described by maximum ventilator support. Infectious components to delivery (i.e., maternal chorioamnionitis) were less consistently predictive, potentially reflecting controversy in the clinical diagnosis of chorioamnionitis [15]. Laboratory markers, although consistently obtained and discussed, do not appear to influence decision-making [16].

Sepsis is exceedingly rare in well-appearing term neonates [17]. In this population, physical examination has an excellent negative predictive value (92\%). However, the definition of a "well-appearing" VLBW neonate is unclear. For example, greater than $90 \%$ of this population experiences respiratory distress at birth [18]. Although this clinical manifestation is often attributed to EOS, it is highly non-specific and more likely attributable to surfactant deficiency and/or retained fetal lung fluid [19]. In fact, epidemiologic studies estimate a risk of earlyonset sepsis of approximately 1\% in extremely 
Table 1 Comparison of short and prolonged initial antibiotic courses

\begin{tabular}{|c|c|c|c|}
\hline Variable & $\leq 3$ days $(n=71)$ & $>5$ days $(n=50)$ & $p$ value \\
\hline \multicolumn{4}{|l|}{ Perinatal factors } \\
\hline Chorioamnionitis & $4(6 \%)$ & $8(16 \%)$ & 0.071 \\
\hline ROM time in hours, median (range) & $0(0-648)$ & $0(0-672)$ & 0.235 \\
\hline \multicolumn{4}{|l|}{ ROM category } \\
\hline$>18 \mathrm{~h}$ to $<7$ days & $12(17 \%)$ & $8(16 \%)$ & 0.306 \\
\hline$>7$ days & $4(6 \%)$ & $6(12 \%)$ & \\
\hline Placental abruption & $8(11 \%)$ & $7(14 \%)$ & 0.780 \\
\hline Preeclampsia & $12(17 \%)$ & $14(28 \%)$ & 0.147 \\
\hline Maternal GBS positive & $6(9 \%)$ & $9(18 \%)$ & 0.442 \\
\hline Infectious component to delivery indication & $50(70 \%)$ & $35(70 \%)$ & 0.960 \\
\hline \multicolumn{4}{|l|}{ Basic demographics } \\
\hline Gestational age in weeks, median (range) & $29(23-33)$ & $25(23-34)$ & $<0.001$ \\
\hline Birth weight in grams, median (range) & $1150(570-1485)$ & $825(350-1490)$ & $<0.001$ \\
\hline Male gender & $43(61 \%)$ & $27(54 \%)$ & 0.472 \\
\hline \multicolumn{4}{|l|}{ Race } \\
\hline White & $37(52 \%)$ & $22(44 \%)$ & 0.488 \\
\hline Black & $30(42 \%)$ & $28(56 \%)$ & \\
\hline Asian & $2(3 \%)$ & $4(8 \%)$ & \\
\hline Ethnicity Hispanic & $2(2.8 \%)$ & $4(8 \%)$ & 0.215 \\
\hline Caesarean section & $53(75 \%)$ & $38(76 \%)$ & 0.865 \\
\hline \multicolumn{4}{|l|}{ Neonatal clinical characteristics } \\
\hline APGAR 5 min of life, median (range) & $7(1-9)$ & $6(0-9)$ & 0.001 \\
\hline CRIB II, median (range) & $7(2-14)$ & $12(3-16)$ & $<0.001$ \\
\hline \multicolumn{4}{|l|}{ Maximum ventilator support in first $48 \mathrm{~h}$} \\
\hline Room air/non-invasive & $18(25 \%)$ & $1(2 \%)$ & $<0.001$ \\
\hline Conventional invasive mechanical & $46(65 \%)$ & $29(58 \%)$ & \\
\hline High-frequency invasive mechanical & $7(10 \%)$ & $20(40 \%)$ & \\
\hline Inotropes in first $48 \mathrm{~h}$ & $4(6 \%)$ & $16(32 \%)$ & 0.001 \\
\hline \multicolumn{4}{|l|}{ Neonatal laboratory characteristics } \\
\hline White blood cell count, median (range) & $7100(1900-28,500)$ & $6400(1400-72,900)$ & 0.068 \\
\hline Absolute neutrophil count $<1000 / \mathrm{mm}^{3}$ & $11(15.5 \%)$ & $12(24 \%)$ & 0.581 \\
\hline Immature: total neutrophil ratio $>0.2$ & $0(0 \%)$ & $3(6 \%)$ & 0.999 \\
\hline
\end{tabular}


Table 1 continued

\begin{tabular}{llll}
\hline Variable & $\boldsymbol{5 3}$ days $(\boldsymbol{n}=\mathbf{7 1})$ & $>\mathbf{5}$ days $(\boldsymbol{n}=\mathbf{5 0})$ & $\boldsymbol{p}$ value \\
\hline Platelet count, median (range) & $184(73-356)$ & $187(91-404)$ & 0.244 \\
\hline
\end{tabular}

$R O M$ rupture of membranes, GBS Group B streptococcus, CRIB II clinical risk index for babies II

Table 2 Multivariate analysis of significant factors associated with a prolonged antibiotic course

\begin{tabular}{|c|c|c|}
\hline Variable & $\begin{array}{l}\text { Odds ratio }(95 \% \\
\text { CI })\end{array}$ & $p$ value \\
\hline $\begin{array}{l}\text { Maximum ventilator } \\
\text { support }\end{array}$ & $3.62(1.42-9.26)$ & 0.007 \\
\hline Chorioamnionitis & $4.14(1.00-17.24)$ & 0.051 \\
\hline Gestational age & $0.88(0.77-1.08)$ & 0.221 \\
\hline 5-min APGAR & $0.83(0.66-1.04)$ & 0.099 \\
\hline
\end{tabular}

preterm neonates [20]. The chasm between this incidence and the rate of prolonged courses of antibiotics in the VLBW population, largely driven by non-specific clinical symptoms in our study, is a source for concern.

Utilization of chorioamnionitis to define risk in VLBW newborns may also be challenged. Recent evidence suggests that the absence of chorioamnionitis can be used to accurately identify low-risk neonates, although these neonates continue to receive prolonged antibiotic therapy in clinical practice, particularly those born at earlier gestational age and lower birthweights $[21,22]$. Clinical chorioamnionitis has consistently been documented to increase the relative risk of EOS, but overall risk remains low [23]. Importantly, the majority of clinical criteria for diagnosing chorioamnionitis have poor sensitivity or are subjective findings [24]. Significant practice variation exists in the diagnosis and management between institutions and providers [25]. Histopathologic diagnosis of chorioamnionitis is rarely available for neonatologists to utilize when analyzing potential infectious risk factors and determining antibiotic initiation and continuation, forcing them to rely on the potentially inaccurate clinical diagnosis. These limitations manifest in our clinical practice, with clinical chorioamnionitis emerging as an independent risk factor justifying prolonged antibiotics in some cases and a non-factor in others.

Biomarkers of neonatal sepsis are an area of intense study. A CBC is universally used as a screening tool for sepsis, but this test lacks sensitivity for EOS. Large multicenter studies document the association between low WBC, low ANC, high I:T ratio, and low platelet counts and increased odds for EOS, but sensitivity for these tests is consistently poor [16]. C-reactive protein (CRP) is an acute-phase reactant known to elevate during both infectious and non-infectious conditions, including those common to the neonatal population, such as ischemia, pneumothorax and chorioamnionitis without invasive fetal disease [26, 27]. It has been extensively studied as a biomarker for neonatal sepsis, and is known to have a 99\% negative predictive value when two CRP values obtained in the first $24-48 \mathrm{~h}$ are normal [19]. Because of this strong negative predictive value, it may have utility in determining when antibiotics can be safely discontinued during a sepsis evaluation. However, physiologic variation and poor sensitivity in the first few days of life, particularly in the first $6-8 \mathrm{~h}$ of life, make its utility as a diagnostic screening test for sepsis low [28]. Hence, recent guidelines do not advise continuation of antibiotic therapy based on serial abnormal values in the absence of cultureconfirmed infection [26]. At our institution, the obtaining and timing of CRPs is variable in VLBW infants, and thus it was not included as a potential laboratory marker for sepsis. Procalcitonin is a well-studied biomarker for sepsis with established normal values in both term and preterm neonates. It has improved sensitivity 
when compared to CRP, and may allow reduced antibiotic duration in neonates $>34$ weeks with suspicion for EOS [29]. Unfortunately, procalcitonin, along with other immunologic mediators such as IL-6, are often not readily available in the clinical setting [30]. While frequently obtained, the lack of reliability of more common laboratory values is reflected in their nonsignificance as key drivers of antibiotic duration in our study.

Limitations of our study include its singlecenter retrospective design, making generalizable application to other sites questionable. Clinically defined conditions, such as chorioamnionitis, may have different criteria and thresholds between sites, resulting in altered application for diagnosis. We also did not carefully examine clinical trajectory; for example, laboratory values were limited to those initially obtained after birth. Blood culture was used as the gold standard for the diagnosis of sepsis; with culture volumes that varied from $0.1 \mathrm{~mL}$ to $1 \mathrm{~mL}$, the clear diagnosis of culture-positive sepsis could have been affected. However, the lack of positive blood cultures in our study population allowed for a clear assessment of other variables impacting antibiotic duration in a diverse, real-world VLBW population.

\section{CONCLUSION}

In conclusion, our study highlights the frequent utilization of non-specific markers of clinical illness to determine antibiotic duration in VLBW neonates. The decision to treat with prolonged antibiotic therapy appears to most consistently rest on the general clinical condition of the neonate. This finding is concerning, given the poor sensitivity of this marker. Both future studies and clinical practice improvements are vital. The development of evidencebased algorithms to objectively estimate the risk of sepsis in preterm neonates based on perinatal risk and clinical status should be prioritized $[11,21]$. Clinical practice initiatives to determine the best allocation of neonatal laboratory monitoring may increase the reliability of blood cultures, or prioritize novel, specific biomarkers for sepsis. Critical evaluation of current clinical practice has the potential to emphasize the high priority of these advances.

\section{ACKNOWLEDGMENTS}

We thank the participants of this study and their families. Jason G. Newland is an Editorial Board Member for Infectious Diseases and Therapy.

Funding. No funding or sponsorship was received for this study or the publication of this article.

Authorship. All named authors meet the International Committee of Medical Journal Editors (ICMJE) criteria for authorship for this article, take responsibility for the integrity of the work as a whole, and have given their approval for this version to be published.

Disclosures. Alexandra C. Charron, Mike A. Carl, Barbara B. Warner, Jason G. Newland and Christopher C. McPherson have nothing to disclose.

Compliance with Ethics Guidelines. This study was performed in compliance with the 1964 Helsinki declaration and its later amendments or comparable ethical standards. The Institutional Review Board of Washington University in St. Louis approved this study and granted a waiver of consent given its retrospective nature.

Data Availability. The datasets generated during and/or analyzed during the current study are not publicly available due to their continued utilization in additional ongoing research, but are available from the corresponding author on reasonable request.

Open Access. This article is distributed under the terms of the Creative Commons Attribution-NonCommercial 4.0 International License (http://creativecommons.org/licenses/ by-nc/4.0/), which permits any noncommercial use, distribution, and reproduction 
in any medium, provided you give appropriate credit to the original author(s) and the source, provide a link to the Creative Commons license, and indicate if changes were made.

\section{REFERENCES}

1. Clark RH, Bloom BT, Spitzer AR, Gerstmann DR. Reported medication use in the neonatal intensive care unit: data from a large national data set. Pediatrics. 2006;117(6):1979-87.

2. Schulman J, Dimand RJ, Lee HC, Duenas GV, Bennett MV, Gould JB. Neonatal intensive care unit antibiotic use. Pediatrics. 2015;135(5):826-33.

3. Stoll BJ, Hansen NI, Bell EF, Walsh MC, Carlo WA, Shankaran $S$, et al. Trends in care practices, morbidity, and mortality of extremely preterm neonates, 1993-2012. JAMA. 2015;314(10):1039-51.

4. Patel SJ, Oshodi A, Prasad P, Delamora P, Larson E, Zaoutis $\mathrm{T}$, et al. Antibiotic use in neonatal intensive care units and adherence with centers for disease control and prevention 12 step campaign to prevent antimicrobial resistance. Pediatr Infect Dis J. 2009;28(12):1047-51.

5. Cotten CM, Taylor S, Stoll B, Goldberg RN, Hansen NI, Sanchez PJ, et al. Prolonged duration of initial empirical antibiotic treatment is associated with increased rates of necrotizing enterocolitis and death for extremely low birth weight infants. Pediatrics. 2009;123(1):58-66.

6. Kuppala VS, Meinzen-Derr J, Morrow AL, Schibler KR. Prolonged initial empirical antibiotic treatment is associated with adverse outcomes in premature infants. J Pediatr. 2011;159(5):720-5.

7. Cotten CM, McDonald S, Stoll B, Goldberg RN, Poole K, Benjamin DK Jr, et al. The association of third-generation cephalosporin use and invasive candidiasis in extremely low birth-weight infants. Pediatrics. 2006;118(2):717-22.

8. Saiman L, Ludington E, Dawson JD, Patterson JE, Rangel-Frausto S, Wiblin RT, et al. Risk factors for Candida species colonization of neonatal intensive care unit patients. Pediatr Infect Dis J. 2001;20(12):1119-24.

9. Clock SA, Ferng YH, Tabibi S, Alba L, Patel SJ, Jia H, et al. Colonization with antimicrobial-resistant gram-negative bacilli at neonatal intensive care unit discharge. J Pediatric Infect Dis Soc. 2017;6(3):219-26.
10. Gibson MK, Wang B, Ahmadi S, Burnham CA, Tarr PI, Warner BB, et al. Developmental dynamics of the preterm infant gut microbiota and antibiotic resistome. Nat Microbiol. 2016;1:16024.

11. Kuzniewicz MW, Puopolo KM, Fischer A, Walsh EM, Li S, Newman TB, et al. A quantitative, riskbased approach to the management of neonatal early-onset sepsis. JAMA Pediatrics. 2017;171(4):365-71.

12. Escobar GJ, Puopolo KM, Wi S, Turk BJ, Kuzniewicz MW, Walsh EM, et al. Stratification of risk of earlyonset sepsis in newborns $>/=34$ weeks' gestation Pediatrics. 2014;133(1):30-6.

13. Newman TB, Puopolo KM, Wi S, Draper D, Escobar GJ. Interpreting complete blood counts soon after birth in newborns at risk for sepsis. Pediatrics. 2010;126(5):903-9.

14. Cordero L, Ayers LW. Duration of empiric antibiotics for suspected early-onset sepsis in extremely low birth weight infants. Infect Control Hosp Epidemiol. 2003;24(9):662-6.

15. Committee on Obstetric Practice. Committee Opinion No. 712: Intrapartum Management of Intraamniotic Infection. Obstet Gynaecol. 2017;130(2):e95-e101.

16. Hornik CP, Benjamin DK, Becker KC, Benjamin DK Jr, Li J, Clark RH, et al. Use of the complete blood cell count in early-onset neonatal sepsis. Pediatr Infect Dis J. 2012;31(8):799-802.

17. Escobar GJ, Li DK, Armstrong MA, Gardner MN, Folck BF, Verdi JE, et al. Neonatal sepsis workups in infants $\geq 2000$ grams at birth: a population-based study. Pediatrics. 2000;106(2 Pt 1):256-63.

18. Stoll BJ, Hansen NI, Bell EF, Shankaran S, Laptook $\mathrm{AR}$, Walsh MC, et al. Neonatal outcomes of extremely preterm infants from the NICHD Neonatal Research Network. Pediatrics. 2010;126(3):443-56.

19. Camacho-Gonzalez A, Spearman PW, Stoll BJ. Neonatal infectious diseases: evaluation of neonatal sepsis. Pediatr Clin North Am. 2013;60(2):367-89.

20. Stoll BJ, Hansen NI, Sanchez PJ, Faix RG, Poindexter BB, Van Meurs KP, et al. Early onset neonatal sepsis: the burden of group B Streptococcal and E. coli disease continues. Pediatrics. 2011;127(5):817-26.

21. Puopolo KM, Mukhopadhyay S, Hansen NI, Cotten CM, Stoll BJ, Sanchez PJ, et al. Identification of extremely premature infants at low risk for earlyonset sepsis. Pediatrics. 2017;140(5):e20170925.

22. Mukhopadhyay S, Puopolo KM. Clinical and microbiologic characteristics of early-onset sepsis 
among very low birth weight infants: opportunities for antibiotic stewardship. Pediatr Infect Dis J. 2017;36(5):477-81.

23. Taylor JA, Opel DJ. Choriophobia: a 1-act play. Pediatrics. 2012;130(2):342-6.

24. Tita AT, Andrews WW. Diagnosis and management of clinical chorioamnionitis. Clin Perinatol. 2010;37(2):339-54.

25. Greenberg MB, Anderson BL, Schulkin J, Norton ME, Aziz N. A first look at chorioamnionitis management practice variation among US obstetricians. Infect Dis Obstetrics Gynecol. 2012;2012:628362.

26. Puopolo KM, Benitz WE, Zaoutis TE, Committee on fetus and newborn, committee on infectious diseases. Management of neonates born at $\leq 346 / 7$ weeks' gestation with suspected or proven earlyonset bacterial sepsis. Pediatrics. 2018;142(6):20182896.
27. Benitz WE. Adjunct laboratory tests in the diagnosis of early-onset neonatal sepsis. Clin Perinatol. 2010;37(2):421-38.

28. Forest JC, Lariviere F, Dolce P, Masson M, Nadeau L. C-reactive protein as biochemical indicator of bacterial infection in neonates. Clin Biochem. 1986;19(3):192-4.

29. Stocker M, van Herk W, El Helou S, Dutta S, Fontana MS, Schuerman F, et al. Procalcitonin-guided decision making for duration of antibiotic therapy in neonates with suspected early-onset sepsis: a multicentre, randomised controlled trial (NeoPIns). Lancet. 2017;390(10097):871-81.

30. Memar MY, Alizadeh N, Varshochi M, Kafil HS. Immunologic biomarkers for diagnostic of earlyonset neonatal sepsis. J Matern Fetal Neonatal Med. 2019;32(1):143-53. 